\title{
International Journal of Sciences
}

\section{Research Article}

\author{
Volume 5 - March 2016 (03)
}

\section{Downregulation of VEGF and KSP Gene Expression Inhibits Proliferation of A549 Cells}

\author{
Xiang-Xu Hu${ }^{1}$, Yue-Hua Wang ${ }^{1}$, Jin-Yu Zhang1
}

${ }^{1}$ Department of Biochemistry and Molecular Biology, Medical College, Qingdao University, 38 Dengzhou Road, Qingdao, China, 266021

\begin{abstract}
To investigate the proliferation and invasion of A549 cell by down-regulating KSP and VEGF gene. Designing and screening the siRNA of targeting VEGF and KSP promoter, transfected into A549 cells with transfetion reagent. According to the difference of transfection mixture the cells were divided into five groups. Western Blotting was used to detect the expression of VEGF and KSP. MTT assay was used to detect the cell proliferation, qPCR was used to measure the expression of VEGF and KSP, the MTT results indicated that the proliferation of the cells was significantly decreased; The results of qPCR showed that the siRNA obviously decreased the expression of VEGF and KSP gene;. The Western Blotting showed that the expression of VEGF and KSP were significantly reduced; The proliferation of the co-transfected cell were inhibited more than the single group transfected cell. These results indicate that co-regulation of VEGF and KSP gene expression more effectively inhibit A549 cell proliferation, better than only regulating VEGF or KSP gene expression.
\end{abstract}

Keywords: A549 cell, siRNA, Gene expression, KSP, VEGF

\section{Intruduction}

Non-small cell lung cancer is a common primary Lung cancer. Laboratory and clinical studies have demonstrated that cancer is a gradual process of long-term accumulation, involving multiple genetic and epigenetic variation, involving decrease of anti-oncogene gene expression and increase of oncogene expression[1]. VEGF is vascular endothelial growth factor gene, cancer cell growth and metastasis depend on angiogenesis, vascular endothelial growth factor is effective angiogenic growth factor[2]. VEGF and VEGFR has become targets for the treatment of cancer.Therefore, VEGF is expected to become a new molecular marker of cancer diagnosis and treatment[3]. KSP is a member of the kinesin family, and plays an important role in early mitosis, formation of the spindle, centrosome separation and chromosome division during mitosis[4]. In addition, KSP is also closely related to tumor development and progression, and highly expressed in many tumor cell lines[5]. It has become an important new target for cancer chemotherapy. Most research focused on siRNA or targeting a single gene, which is an effective way to control single-gene diseases[6]. There are various compensatory mechanisms and complex signal transduction pathway in cancer cell, therefore the effect becomes poor of siRNA controlling cancer[7]. We think that downregulate $K S P$ and $V E G F$ will have better effect in cancer therapy. In this study, we first designed and screened siRNA of cancer gene VEGF and KSP. Then, transfected siRNA into A549 cell respectively, detected its impact on proliferation, invasion, expression of the target gene. Finally,

This article is published under the terms of the Creative Commons Attribution License 4.0

Author(s) retain the copyright of this article. Publication rights with Alkhaer Publications.

Published at: http://www.ijsciences.com/pub/issue/2016-03/

DOI: 10.18483/ijSci.963; Online ISSN: 2305-3925; Print ISSN: 2410-4477 
co-transfected to explore the application value cotroling proliferation and invasionon of A549 cell.

\section{Materials and Methods}

\subsection{Cell cultures}

A549 cells were acquired from the Biochemistry experiment center of Qingdao Medical College. The cells were maintained in Roswell Park Memorial Institute medium (RPMI-1640), containing $10 \%$ fetal bovine serum, 100U/ml penicillin and $100 \mu \mathrm{g} / \mathrm{ml}$ strepto-mycin, and incubated at $37^{\circ} \mathrm{C}$ in a humidified atmosphere with $5 \% \mathrm{CO}_{2}$. Cells in exponential growth phase were used throughout the experiments.

\section{2 siRNA sequences design}

The sequences of the RNAs oligonucleotides were designed as follows: VEGF siRNA, 5'-GGAGUACCCUGAUGAGAUCTT-3' (sense) and 5'-GAUCUCAUCAGGGUACUCCTT -3' (antisense); KSP siRNA,

5'-CUGAAGACCUGAAGACAAUTT-3' (sense) and 5'-AUUG UCUUCAGGUCUUCAGTT-3' (antisense); Negative control, 5'-UUCUCCGAACGUGUC ACGUTT-3' (sense) and 5'-ACGUGACACGUU CGGAGAATT-3' (antisense); The selected sequences did not share any homology with any of the known mRNA databases.

\subsection{Transfection of RNAs oligonucleotides}

The siRNAs were transfected into cultured cells at $65 \%$ confluence which had been seeded in 6-well plates $24 \mathrm{~h}$ earlier. For transfection, Invitrogen ${ }^{\mathrm{TM}}$ Lipofectamine 2000 reagent (Thermo Fisher Scientific, Inc.) was used as RNAs carrier. Five groups of cells were established as follows: CT (control group), NC (non-specific transfection group), VEGF groups, KSP groups, VEGF+KSP groups (co-transfection group). The cells in the CT group were treated with Opti-MEM (Thermo Fisher Scientific, Inc.), while the cells in the other groups were treated with Lipofectamine 2000-Opti-MEM. At 5-6 $\mathrm{h}$ post-transfect, the medium was replaced with $10 \%$ serum-supplemented RPMI-1640, and the cells were incubated for additional 24-96 h. Next, the cells were harvested by centrifugation, rinsed with phosphate buffered saline, and subjected to total RNA or protein extraction.

\subsection{Cell proliferation assay}

Cell viability was examined by MTT assay (Roche Diagnostics). For the experiment, A549 cells were seeded in a 96-well plate (Corning Life Sciences, New York, NY, USA) at a density of $5 \times 10^{3}$ cells/well, and transfected with siRNA and then we need to change the medium six hours after transfection. At 24 , 48 and $72 \mathrm{~h}$ post-transfection, MTT $(5 \mathrm{mg} / \mathrm{ml})$ was added to the cells, which were incubated at $37^{\circ} \mathrm{C}$ for additional $4 \mathrm{~h}$. Next, the supernatant was removed, and $150 \mu \mathrm{l}$ dimethyl sulfoxide (Sigma-Aldrich) was added to each well, followed by 10-min agitation. The absorbance of each well at $490 \mathrm{~nm}$ was measured using an ELISA plate reader (Thermo Multiskan MK3; Thermo Fisher Scientific, Shanghai, China). Quintuplicate wells were used for each experimental condition, and all the experiments were repeated at least three times.

\subsection{Total RNA extraction and quantitative polymerase chain reaction (qPCR)}

Total RNA was isolated from the cells using Invitrogen TM TRIzol reagent (Thermo Fisher Scientific, Inc.), and cDNA was prepared with First Strand cDNA Synthesis Kit (Roche Diagnostics, Basel, Switzerland), according to the manufacturer's instructions. Next, the cDNA amplified by qPCR, using the following primers (Shanghai Sangon Biotech Co., Ltd., Shanghai, China): VEGF, forward 5'-TGCCCACTGAGGAGTCCAAC-3'and reverse 5'-TGGTTCCCGAAACGCTGAG-3'; KSP, forward 5'-CCCCGTAACAAGAGAGGAGTG -3'and reverse 5'-TCCTTTTTGCTGCCCCCTTT-3'; The reaction conditions were as follows: $94^{\circ} \mathrm{C}$ for $30 \mathrm{sec}$, followed by 40 cycles of annealing at 60 or $72^{\circ} \mathrm{C}$ (KSP or VEGF respectively) for $30 \mathrm{sec}$, and extension at $72^{\circ} \mathrm{C}$ for $30 \mathrm{sec}$. qPCR amplification was performed on a Corbett Rotor-Gene 3000 (Qiagen, Inc., Valencia, CA, USA), using FastStart Universal SYBR Green Master (Rox) (Roche Diagnostics). 
Each RNA sample was analyzed in triplicate.

\subsection{Western blot analysis}

The western blot was used to detected the expression of VEGF, KSP and bcl2. Total protein content was extracted from the cells with RIPA lysis buffer (Beyotime Institute of Biotechnology, Nantong, China). The protein concentration in the cell lysates was determined with A280 (nm) ultraviolet absorption method. The samples were separated by SDS-PAGE using 10\% polyacrylamide gels (Beijing Solarbio Science \& Technology Co., Ltd., Beijing, China) and then transferred to PVDF membranes. Membranes were blocked for $1 \mathrm{~h}$ with blocking buffer (5\% BSA or $5 \%$ non-fat milk) at room temperature. Subsequently, primary antibodies were added to the membrane and incubated overnight at $4^{\circ} \mathrm{C}$. Antibodies were diluted 1:1,000. while the mouse monoclonal antibodies against GAPDH was diluted 1:2,000. Following 3 washes of 5 min each with TBST, the membrane were incubated with secondary antibody for $1 \mathrm{~h}$ at room temperature, following 3 washes of $5 \mathrm{~min}$ each with TBST, and then proteins were visualized using the enhanced chemiluminescence method, and protein expression was quantified with a Gel EDAS 293 analysis system.

\subsection{Statistical analysis}

All of the data were used statistical software SPSS17.0 do statistical analysis, and the results expressed in $\overline{\mathrm{x}} \pm \mathrm{s}$, using the single factor ANOVA method do multiple comparison and the LSD methodhe do comparison between two groups.

\section{Results}

2.1 Cell morphology was observed in all groups after transfection $48 \mathrm{~h}$

cells in NC and CT groups has a good shape, fusiform. The difference was not significant. KSP and VEGF groups cells increased apoptosis, cell rounding, and the two groups has no significant difference of apoptosis number. the number of apoptotic cells and round cells of KSP+VEGF groups was significant increased. Comparing VEGF+KSP group with KSP or VEGF group, the difference of was also significant .Figure 1.

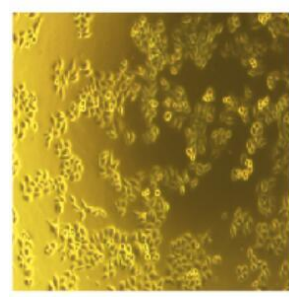

CT

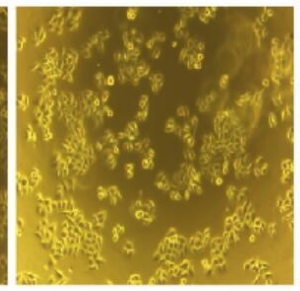

NC

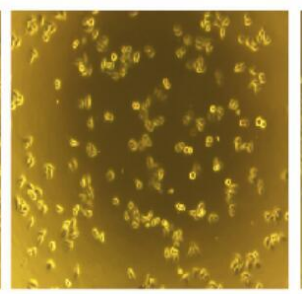

KSP

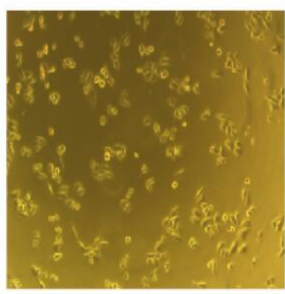

VEGF

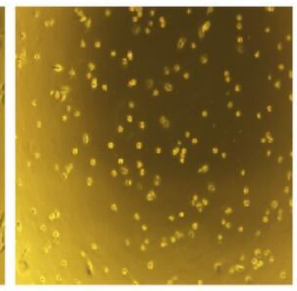

KSP+VEGF

Figure 1. Morphology of each groups after transfection $48 \mathrm{~h}$

\section{2 siRNA effectively inhibited the proliferation of A549 cells.}

The results showed in Table 2. When siRNA independent and co-transfected A549 cells for 24h, $48 \mathrm{~h}, 72 \mathrm{~h}$, the optical density of KSP group or VEGF group was significantly lower than that of CT group $(p<0.05)$, while the optical density of KSP+VEGF group was much lower. Comparing VEGF or KSP group with KSP+VEGF group, the difference of ODs was also significant $(\mathrm{p}<0.05)$. However, there was no difference between NC group and CT group, on the other words, the difference was not significant $(\mathrm{p}>0.05)$. 
Table 2. cell proliferation rate $(n=5, x \pm s)$

\begin{tabular}{llll}
\hline GROUP & $24 \mathrm{~h}$ & $48 \mathrm{~h}$ & $72 \mathrm{~h}$ \\
\hline $\mathrm{CT}$ & $1.131 \pm 0.044$ & $1.343 \pm 0.031$ & $1.448 \pm 0.023$ \\
$\mathrm{NC}$ & $1.124 \pm 0.034$ & $1.333 \pm 0.013$ & $1.427 \pm 0.028$ \\
VEGF & $0.834 \pm 0.008^{* *}$ & $0.654 \pm 0.017 * *$ & $0.635 \pm 0.012^{* *}$ \\
KSP & $0.732 \pm 0.035^{* *}$ & $0.623 \pm 0.012^{* *}$ & $0.521 \pm 0.015^{* *}$ \\
VEGF+KSP & $0.623 \pm 0.066^{*}$ & $0.565 \pm 0.032^{*}$ & $0.433 \pm 0.069^{*}$ \\
\hline
\end{tabular}

\section{3 the protein and miRNA expression levels after transfection}

The results showed that the expressions of KSP, VEGF, bcl2 protein and mRNA in KSP, VEGF and $\mathrm{KSP}+\mathrm{VEGF}$ groups were significantly lower than the CT and NC groups $(\mathrm{p}<0.05)$; The expressions of
mRNA and protein in VEGF+KSP groups were significantly lower than KSP or VEGF groups $(\mathrm{p}<0.05)$. Table 3.

Table 3. The protein and miRNA expression levels of each groups

\begin{tabular}{|l|l|l|l|l|l|}
\hline \multirow{2}{*}{ GROUP } & \multicolumn{4}{|l|}{ VEGF } & \multicolumn{2}{l|}{ KSP } & BCL2 \\
\cline { 2 - 6 } & protein & mRNA & protein & mRNA & mRNA \\
\hline CT & $1.25 \pm 0.01$ & $1.10 \pm 0.01$ & $1.50 \pm 0.06$ & $1.12 \pm 0.02$ & $0.94 \pm 0.05$ \\
\hline NC & $1.32 \pm 0.01$ & $0.97 \pm 0.03$ & $1.46 \pm 0.05$ & $0.85 \pm 0.10$ & $0.91 \pm 0.05$ \\
\hline VEGF & $0.89 \pm 0.01$ & $0.10 \pm 0.01$ & - & - & $0.72 \pm 0.05$ \\
\hline KSP & - & - & $1.08 \pm 0.05$ & $0.13 \pm 0.03$ & $0.69 \pm 0.02$ \\
\hline VEGF+KSP & $0.51 \pm 0.01$ & $0.06 \pm 0.01$ & $0.83 \pm 0.04$ & $0.07 \pm 0.01$ & $0.50 \pm 0.01$ \\
\hline
\end{tabular}

\section{Discussion}

Nearly half a century, with the development of industrialization, lung cancer incidence and mortality has been on a clear upward trend ${ }^{[8]}$, but there is no fully effective drug to treat lung cancer. More and more studies show that siRNA technology can effectively inhibit the proliferation of cancer cells ${ }^{[9]}$. Therefore, finding an effective siRNAs target site may be one of the most popular cancer treatment.

In recent years studies have shown that lung cancer development, metastasis and prognosis had closely related to angiogenesis ${ }^{[10]}$. Some studies suggest that immortalized tumor cell formation and angiogenesis are closely related. In recent years, we found that many of angiogenesis factors, including $V E G F$ is a new discovery of the role of the current strong and specific vascular regulatory factors ${ }^{[11]}$. $V E G F$, also known as vascular permeability factor, vascular endothelial cell proliferation, vascular basement membrane hydrolysis and build stronger role, and high specificity, tumor angiogenesis is induced strong and specific role of regulator ${ }^{[12]}$. Transfection of targeting VEGF siRNAs, which can effectively lower mRNA and Protein levels. With the reduced VEGF expression, $\mathrm{Bcl} 2$ expression was significantly reduced, the degree of apoptosis was significantly increased.

KSP has conserved ATP enzyme domain and dynamic domains, it play an important role in formation of a bipolar spindle and separation processes in mitotic metaphase ${ }^{[13]}$. Studies have shown, the KSP siRNA can lead to the generation of a single star spindle promote mitotic arrest in $\mathrm{M}$ phase and induce apoptosis of tumor cells ${ }^{[14]}$. Studies 
have shown that, KSP is highly expressed in human malignant proliferation of tissue cells, the expression level of cell proliferation and mitosis and tumor proportion of students Growth rate correlated. KSP protein involved in cell mitosis ${ }^{[15]}$, the body's normal proliferation of cells, there are also the expression of $K S P$, but its expression level was significantly lower than the malignant tissue; the body's normal differentiation and maturation of cells and tissues, such as nerve cells were not detected in the KSP expression $^{[16]}$. Thus, inhibition of KSP Can inhibit cell mitosis, the cells stop proliferating. In this study, the use of siRNA to inhibit KSP expression, apoptosis was significantly increased, suggesting that reduced expression of KSP can inhibit cell proliferation and induce apoptosis.

In most solid malignant tumors, including breast expression, neuroblastoma and lung cancer, $V E G F$ and KSP were raised, related to the malignant behavior of tumor. Therefore, more and more research will regard $V E G F$ and $K S P$ as a target site for cancer treatment ${ }^{[17]}$. There is no simultaneous siRNAs targeting VEGF and $K S P$ in non-small cell lung cancer cell proliferation, apoptosis, invasion of research reports. Therefore, this study show that synthesis siRNAs and transfection of A549 cells to inhibiting the expression of VEGF and KSP genes. Results of this study showed that double interference group apoptosis rate was significantly higher than the single interference group.Similarly, VEGF and KSP expression in a co-interference were significantly lower than KSP or VEGF groups.

In summary, siRNA tramsfection reduced expression of $V E G F$ and KSP gene, the effect of inhibiting the growth of lung cancer cells was significantly higher than any of the individual down-regulated genes; $V E G F$ and $K S P$ can be used as both an important target for cancer therapy. Joint downregulation of $K S P$ and $V E G F$ can be used as a reference for breast cancer treatment. This research results provides an experimental basis to multiple gene silencing to inhibition of tumor cell proliferation.

\section{REFERENCES}

1) Haijun Yu,Yonglong Zou, Lei Jiang, Qi Yin, Xinyu He, Lingli Chen, Zhiwe Zhang, Wangwen Gu,Yaping Li. Induction of apoptosis in non-small cell lung cancer by downregulation of MDM2 using $\mathrm{pH}$-responsive PMPC-b-PDPA/siRNA complex nanoparticles[J]. Biomaterials,2013,3411.

2) Stelios Florinas, Jaesung Kim,Kihoon Nam, Margit M. Janát-Amsbury, Sung Wan Kim. Ultrasound-assisted siRNA delivery via arginine-grafted bioreducible polymer and microbubbles targeting VEGF for ovarian cancer treatment[J]. Journal of Controlled Release, 2014.

3) Hyun Ah Kim, Kihoon Nam, Sung Wan Kim. Tumor targeting RGD conjugated bio-reducible polymer for VEGF siRNA expressing plasmid delivery[J]. Biomaterials, 2014.

4) Basso Andrea D, Liu Ming,Gray Kimberly, Tevar Seema,Lee Suining, Liang Lianzhu, Ponery Abdul, Smith Elizabeth B, Monsma Frederick J, Yu Tao, Zhang Yonglian, Kerekes Angela D, Esposite Sara, Xiao Yushi, Tagat Jayaram R, Hicklin Daniel J, Kirschmeier Paul. SCH 1473759, a novel Aurora inhibitor, demonstrates enhanced anti-tumor activity in combination with taxanes and KSP inhibitors. [J]. Cancer Chemotherapy and Pharmacology, 2011, 684.

5) Dhananjay Jere, Hu-Lin Jiang, You-Kyoung Kim, Rohidas Arote, Yun-Jaie Choi, Cheol-Heui Yun, Myung-Haing Cho, Chong-Su Cho. Chitosan-graft-polyethylenimine for Akt1 siRNA delivery to lung cancer cells[J]. International Journal of Phar maceutics,2009,3781.

6) Tai Jian-dong, Wang Guang-yi, Liu Tong-jun, Wang Lei,Li Feng. [Effect of siRNA targeting c-Myc and VEGF on human colorectal cancer cells]. [J]. Zhonghua Wei-chang Waike Zazhi, 2009, 125.

7) João Conde, Furong Tian,Yulán Hernández, Chenchen Bao, Daxiang Cui, Klaus-Peter Janssen, M. Ricardo Ibarra, Pedro V. Baptista, Tobias Stoeger, Jesús M. de la Fuente. In vivo tumor targeting via nanoparticle-mediated therapeutic siRNA coupled to inflammatory response in lung cancer mouse models[J]. Biomaterials,2013,3431.

8) Yijie Chen, Hongchen Gu, Ding Sheng-Zi Zhang,Fan Li, Tengyuan Liu, Weiliang Xia. Highly effective inhibition of lung cancer growth and metastasis by systemic delivery of siRNA via multimodal mesoporous silica-based nanocarrier[J]. Biomaterials,2014, 3538.

9) Judge, Adam D, Robbins, Marjorie, Tavakoli, Iran, Levi, 
Jasna,Hu, Lina, Fronda, Anna, Ambegia, Ellen, McClintock, Kevin, MacLachlan, Ian. Confirming the RNAi-mediated mechanism of action of siRNA-based cancer therapeutics in mice[J]. Journal of Clinicallnvestigation,2009,1193.

10) Lu Han, Cui Tang, Chunhua Yin. Oral delivery of shRNA and siRNA via multifunctional polymeric nanoparticles for synergistic cancer therapy[J]. Biomaterials, 2014, 3515.

11) Zhenzhen Yang,Bai Xiang,Dawen Dong, Zhanzhang Wang, Jingquan Li, Xianrong Qi. Dual Receptor-Specific Peptides Modified Liposomes as VEGF siRNA Vector for Tumor-Targeting Therapy[J]. Current Gene Therapy, 2014, 144.

12) Kun Liu, Honglin Chen, Qingsheng You, Hai Shi, Zhiwei Wang. The siRNA cocktail targeting VEGF and HER2 inhibition on the proliferation and induced apoptosis of gastric cancer cell[J]. Molecular and Cellular Biochemistry, 2014, 3861-2.

13) Xu Li, Yijie Chen, Mingqi Wang, Yongjie Ma, Weiliang Xia, Hongchen $\mathrm{Gu}$. A mesoporous silica
nanoparticle-PEI-Fusogenic peptide system for siRNA delivery in cancer therapy[J]. Biomaterials, 2013, 344.

14) Joung-Pyo Nam,Kihoon Nam, Simhyun Jung, Jae-Woon Nah, Sung Wan Kim. Evaluation of dendrimer type bio-reducible polymer as a siRNA delivery carrier for cancer therapy[J]. Journal of Controlled Release, 2015,209.

15) Emanuele Marra, Fabio Palombo, Gennaro Ciliberto, Luigi Aurisicchio. Kinesin spindle protein SiRNA slows tumor progression[J]. J. Cell. Physiol.,2012, 2281.

16) Kim Young-Dong, Park Tae-Eun, Singh Bijay, Maharjan Sushila, Choi Yun-Jaie, Choung Pill-Hoon, Arote Rohidas B, Cho Chong-Su. Nanoparticle-mediated delivery of siRNA for effective lung cancer therapy. [J]. Nanomedicine (London, England), 2015, 107.

17) Mei-Hua Qu, Rui-Fang Zeng,Shi Fang, Qiang-Sheng Dai, He-Ping Li, Jian-Ting Long. Liposome-based co-delivery of siRNA and docetaxel for the synergistic treatment of lung cancer[J]. International Journal of Pharmaceutics, 2014, 4741-2. 